\title{
Nonlinear random wave field in shallow water: variable Korteweg-de Vries framework
}

\author{
A. Sergeeva, E. Pelinovsky, and T. Talipova \\ Institute of Applied Physics, Nizhny Novgorod, Russia
}

Received: 27 September 2010 - Revised: 23 November 2010 - Accepted: 24 November 2010 - Published: 3 February 2011

\begin{abstract}
The transformation of a random wave field in shallow water of variable depth is analyzed within the framework of the variable-coefficient Korteweg-de Vries equation. The characteristic wave height varies with depth according to Green's law, and this follows rigorously from the theoretical model. The skewness and kurtosis are computed, and it is shown that they increase when the depth decreases, and simultaneously the wave state deviates from the Gaussian. The probability of large-amplitude (rogue) waves increases within the transition zone. The characteristics of this process depend on the wave steepness, which is characterized in terms of the Ursell parameter. The results obtained show that the number of rogue waves may deviate significantly from the value expected for a flat bottom of a given depth. If the random wave field is represented as a soliton gas, the probabilities of soliton amplitudes increase to a high-amplitude range and the number of large-amplitude (rogue) solitons increases when the water shallows.
\end{abstract}

\section{Introduction}

Rogue or freak waves, which rapidly and surprisingly appear and disappear on the sea surface, are widely observed in various areas of the World Ocean in both open seas and coastal zones; such data is collected in Lavrenov (2003), Didenkulova et al. (2006), Liu (2007), Kharif et al. (2009). Several physical mechanisms are suggested to explain the rogue wave phenomenon (Dysthe et al., 2008; Kharif et al., 2009): (i) dispersive and geometrical focusing of wave packets propagating with different speeds in different directions; (ii) nonlinear mechanisms due to the BenjaminFeir instability and wave-wave interaction; (iii) wave

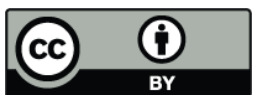

Correspondence to: A. Sergeeva (a.sergeeva@hydro.appl.sci-nnov.ru) interaction with a variable bottom, with currents and wind flow. These physical mechanisms are the subject of intense study in frameworks of various theoretical models of water waves and also through simulations in laboratory tanks; see for instance Kharif et al. (2009).

For practical purposes it is necessary to compute the probability of rogue wave occurrence. Usually, the distribution functions of the wind wave field including extreme characteristics are calculated under assumption of the non-Gaussian properties of a process using the nonlinear perturbation technique; see for instance Dysthe et al. (2008). The nonlinear effect of the Benjamin-Feir instability for narrow-band wave packets over deep water is incorporated in the statistical theory of the weak (wave) turbulence. It leads to the non-Gaussian statistics of wind waves with third and fourth moments (skewness and kurtosis) depending on the Benjamin-Feir index which represents the ratio of nonlinearity to dispersion (Janssen, 2003; Mori and Janssen, 2006). As a result, the probability of rogue wave occurrence increases due to the Benjamin-Feir instability and this effect has been verified experimentally in a long wave flume (Onorato et al., 2006; Shemer and Sergeeva, 2009; Shemer et al., 2010).

The influence of the combined effects of nonlinear dispersive focusing of unidirectional random waves over shallow water (where the Benjamin-Feir instability is absent) on the probability of freak wave appearance has been studied within the Korteweg-de Vries equation (Pelinovsky and Sergeeva, 2006). In this case the wave field remains nonGaussian, and the skewness and kurtosis are controlled by the Ursell parameter, which is proportional to the ratio of nonlinear over dispersive effects.

The bottom topography variability in the coastal zone can also increase the rogue wave probability due to the effect of geometric focusing, which leads to a spontaneous appearance of caustics and focal points (see for instance Lavrenov, 2003; Kharif et al., 2009). When an unidirectional

Published by Copernicus Publications on behalf of the European Geosciences Union. 
wave propagation over variable bottom is concerned, the wave characteristics are non-Gaussian; the runup of random long waves on a plane beach is one of these examples. The statistical moments for this case are studied theoretically in Didenkulova et al. $(2008,2011)$ and experimentally in Huntley et al. (1977).

The influence of the bathymetry non-uniformity on kurtosis and the probability of freak waves has been investigated within the framework of the nonlinear Schrödinger equation in Zeng and Trulsen (2010). They found that after a depth variation, the propagating wave train may need a considerable distance to relax towards the new equilibrium state, which corresponds to the new depth. As a result, kurtosis and the number of freak waves may be significantly different from the values expected for the constant depth environment. This analysis is limited by the narrow-band dispersive wave packet under conditions at intermediate depth.

The main goal of the present paper is to investigate the effect of variable bathymetry on the unidirectional random wave propagation in shallow water. The theoretical model is based on the variable-coefficient Korteweg-de Vries equation (Sect. 2). If the wave field is represented by an ensemble of solitons with random amplitudes and phases, the distribution function of soliton heights is shown to grow in highamplitude limit (the "tail" of the distribution function), and the number of "freak" solitons increases when the depth becomes less. The results of numerical simulations on propagation of initially random wave field under condition when water shallows, are given in Sect. 3. It is emphasized within this framework, that the freak wave probability should increase when depth decreases. The entrance of random waves to a deeper water region leads to the reduction of the number of freak waves, as discussed in Sect. 4. The results obtained are summarized in Sect. 5.

\section{The Korteweg-de Vries model}

The Korteweg-de Vries equation is a basic equation for describing a unidirectional propagation of weakly-nonlinear weakly-dispersive waves in shallow waters. In the case of a slowly variable depth when the wave reflection from the bottom slopes can be neglected, the variable-coefficient Korteweg-de Vries equation has been derived by Ostrovsky and Pelinovsky (1970), Johnson (1973). It can be written as

$\sqrt{g h(x)} \frac{\partial \eta}{\partial x}+\frac{3 \eta}{2 h}\left(\frac{h(x)}{h_{0}}\right)^{-1 / 4} \frac{\partial \eta}{\partial s}+\frac{h(x)}{6 g} \frac{\partial^{3} \eta}{\partial s^{3}}=0$,

where the surface elevation $\zeta(x, t)$ and the time $t$ are introduced as follows,

$\zeta(x, t)=\eta(x, t)\left[\frac{h(x)}{h_{0}}\right]^{-1 / 4}, \quad s=\int_{x_{0}}^{x} \frac{d y}{\sqrt{g h(y)}}-t$, $s$ is the time in the reference system of coordinates, $x$ is the coordinate directed onshore, $h_{0}=h\left(x_{0}\right)$ is the depth of the deepest tide-gauge where an initial condition is defined.

Equation (1) is solved numerically for the periodic domain on $s$ with the period $T, \zeta(0, x)=\zeta(T, x)$. Two integrals of Eq. (1) are conserved,

$$
\int_{0}^{T} \eta(x, s) d s=\mathrm{const}, \quad \int_{0}^{T} \eta^{2}(x, s) d s=\mathrm{const} .
$$

The pseudo-spectral method for time derivation and the Crank-Nicolson scheme for space discretization are used, see details in Fornberg (1998). The solution of (1) is calculated in the discrete Fourier space for spectral amplitudes and defined through the Fourier transform $C(\omega)=$ FFT $\{\eta(s, x)\}$, when the nonlinear part of (1) is calculated as $C_{\text {non }}(\omega)=$ FFT $\left\{\eta^{2}(s, x) / 2\right\}$

$$
\begin{gathered}
C(\omega, x+\Delta x)=C(\omega, x-\Delta x) \frac{1+i \beta \omega^{3} \Delta x}{1-i \beta \omega^{3} \Delta x} \\
-\alpha \operatorname{FFT}\left\{\frac{1}{2} \eta^{2}(s, x)\right\} \frac{i \omega \Delta x}{1-i \beta \omega^{3} \Delta x}, \\
\alpha=\frac{3}{2 h \sqrt{g h}}\left(\frac{h}{h_{0}}\right)^{-1 / 4}, \quad \beta=\frac{h}{6 g \sqrt{g h}} .
\end{gathered}
$$

The initial conditions for (1) at $x_{0}=0$ were defined as a superposition of independent Fourier harmonics with random phases $\varphi$ uniformly distributed within the interval $\left[\begin{array}{ll}0 & 2 \pi\end{array}\right]$

$\zeta(s, x=0)=\eta(s, x=0)=\sum_{n} \sqrt{2 S\left(\omega_{n}\right)} \cos \left(\omega_{n} s+\varphi_{n}\right)$.

The total duration of each realization is about 30 characteristic wave periods corresponding to the typical swell period $T_{0}=15 \mathrm{~s}$ and sample frequency $f=5.7 \mathrm{~Hz}(N=1024$ data points are used for the discretization in time $s$ ). The spatial discretization in (4) is much less then a wave length and is equal to $\Delta x=0.375 h_{0}$. The spectrum of water waves at initial location was assumed to have a Gaussian shape

$S(\omega)=A^{2} \exp \left[-\left(\frac{\omega-\omega_{0}}{\delta \omega}\right)^{2}\right]$,

where $A$ is the characteristic wave amplitude, and the spectral width is set at $\delta \omega / \omega_{0}=0.36$. In the numerical experiments two values of the initial steepness parameter $\varepsilon=A k_{0}$ are considered: $1 \times 10^{-2}$ and $5 \times 10^{-2}$, where the characteristic wave length, defined through the velocity of long waves $\lambda_{0}=2 \pi / k_{0}$ on the depth of $10 \mathrm{~m}$ was about $150 \mathrm{~m}$, providing the condition of a weak dispersion of the waves. Although the initial irregular wave field is supposed to be Gaussian due to the Central Limit Theorem, in numerical experiments some deviations from the Gaussian process naturally occur due to the finiteness of realizations. 
The following variable depth profile is employed in the numerical experiments:

$$
\frac{h(x)}{h_{0}}=\frac{1+h_{1} / h_{0}}{2}-\frac{1-h_{1} / h_{0}}{2} \tanh (x / L) .
$$

Here the initial depth $h_{0}$ is set to $10 \mathrm{~m}$, and the final depth was $h_{1}=5 \mathrm{~m}$. The length of the transitional zone, $L$, varies from $30 h_{0}$ to $120 h_{0}$ (2-8 wavelengths).

The main goal of the numerical simulations is to find the wave field statistical moments. The first two statistical moments can be easily found analytically with the use of (3). In particular, the first moment is

$$
\begin{aligned}
m_{1}(x) & =\int_{0}^{T} \zeta(x, s) d s \\
= & {\left[\frac{h(x)}{h_{0}}\right]^{-1 / 4} \int_{0}^{T} \eta(x, s) d s \sim\left[\frac{h(x)}{h_{0}}\right]^{-1 / 4} m_{1}\left(x_{0}\right), }
\end{aligned}
$$

Meanwhile, at the initial location $m_{1}\left(x_{0}\right)=0$; therefore the mean water level does not change with distance. The second moment reads

$$
\begin{aligned}
& m_{2}(x)=\sigma^{2}(x)=\int_{0}^{T} \zeta^{2}(x, s) d s \\
& =\left[\frac{h(x)}{h_{0}}\right]^{-1 / 2} \int_{0}^{T} \eta^{2}(x, s) d s \sim\left[\frac{h(x)}{h_{0}}\right]^{-1 / 2} m_{2}\left(x_{0}\right) .
\end{aligned}
$$

The characteristic wave amplitude (defined here as half of the significant wave height) is proportional to $\sigma(x)$ as $A_{\mathrm{s}}(x)=2 \sigma(x)$. It varies with depth according to Green's law $h^{-1 / 4}(x)$. The skewness and kurtosis defined as

$\mu_{3}(x)=\frac{m_{3}(x)}{\sigma^{3}(x)}, \quad \mu_{4}(x)=\frac{m_{4}(x)}{\sigma^{4}(x)}$

can not be obtained analytically, and are computed by means of numerical simulations.

Simple analytical results can be achieved for the case when the random field constitutes a "solitonic gas". The soliton turbulence within the framework of the Korteweg-de Vries equation has specific features related to integrability: solitons preserve their individuality, and interactions between them are elastic, and thus do not change soliton amplitudes but alter phases, see Zakharov (1971), Salupere et al. (1996, 2002, 2003a, b), and textbooks Novikov et al. (1980), Drazin and Johnson (1996).

Assuming the random solitons to be located far from each other, the soliton transformation in the coastal zone (shoaling) may be analysed independently for every solitary wave. This dynamics is known to depend on the ratio of nonlinearity length with respect to the width of the transition zone (Tappert and Zabusky, 1971; Pelinovsky, 1971; Nakoulima et al., 2005). If the solitary wave amplitude is large, the soliton quickly adapts to the local depth, so that its new length coincides with the local conditions. In this case the soliton amplitude is proportional to $h^{-1}(x)$; see Ostrovsky and Pelinovsky (1970), Johnson (1973). If the solitary wave has small amplitude, and thus, large wave length, it transforms as a linear wave, and its amplitude is proportional to $h^{-1 / 4}(x)$; see Pelinovsky (1971), Nakoulima et al. (2005). As a result, the distribution of soliton amplitudes changes with depth, and the portion of largeamplitude waves enhances more significantly than the portion of smaller-amplitude solitons.

Thus, the nonlinear property of the soliton field tends to increase the probability of occurrence of large-amplitude waves (freak waves). Of course, this analysis is limited by the low-density "soliton gas" representation of the wave field. In general, the wave field is represented by a superposition of sinusoidal waves with random phases (4), and the statistical moments are calculated numerically, as reported below.

\section{Shoaling of random nonlinear waves}

Let us suppose the region where the initial condition is defined to be far from the transitional zone. Then the Korteweg-de Vries equation for a constant depth may be employed. It is demonstrated in Pelinovsky and Sergeeva (2006) that during the nonlinear wave evolution, the Gaussianity of the process breaks down after a transitional period. Later on, a steady state is established (regarding statistical characteristics), which differs from the Gaussian state and is characterized by a stronger wave asymmetry and kurtosis, when the Ursell parameter grows. The Ursell parameter defines the ratio of nonlinearity $A_{\mathrm{s}} / h(x)$ with respect to dispersion $\left[k_{0} h(x)\right]^{2}$,

$U r(x)=\frac{A_{\mathrm{s}}(x)}{k_{0}^{2}(x) h^{3}(x)}=\frac{2 \sigma(0) g h_{0}^{1 / 4}}{\omega_{0}^{2} h(x)^{9 / 4}}$,

where $g$ is the gravity acceleration. Strictly speaking, the Ursell parameter is defined for a sinusoidal wave with frequency $\omega_{0}$.

Our simulations exhibit the spectrum broadening due to the nonlinearity effect; it also becomes asymmetrical, see Fig. 1 (see also, Pelinovsky and Sergeeva, 2006) and a second harmonic peak is observed. For estimating the Ursell parameter, we used the value of $\omega_{0}$, obtained on the basis of the initial Gaussian spectrum (6) through the spectral moments,

$\omega_{0}=\frac{\int \omega S(\omega) d \omega}{\int S(\omega) d \omega}$.

In the numerical simulations we start with the steepness parameter $\varepsilon=k_{0} A_{\mathrm{S}}=5 \times 10^{-3}$ and the corresponding Ursell 


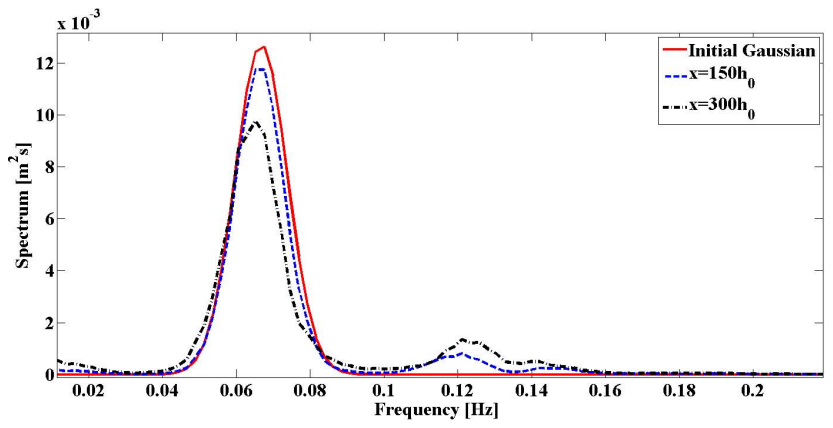

Fig. 1. Power spectra of surface elevation at $x=150 h_{0}$ and at $x=300 h_{0}$ for $U r=0.4$. Red solid line corresponds to the initial Gaussian shaped spectrum. Constant depth $h_{0}=10 \mathrm{~m}$.

(a)
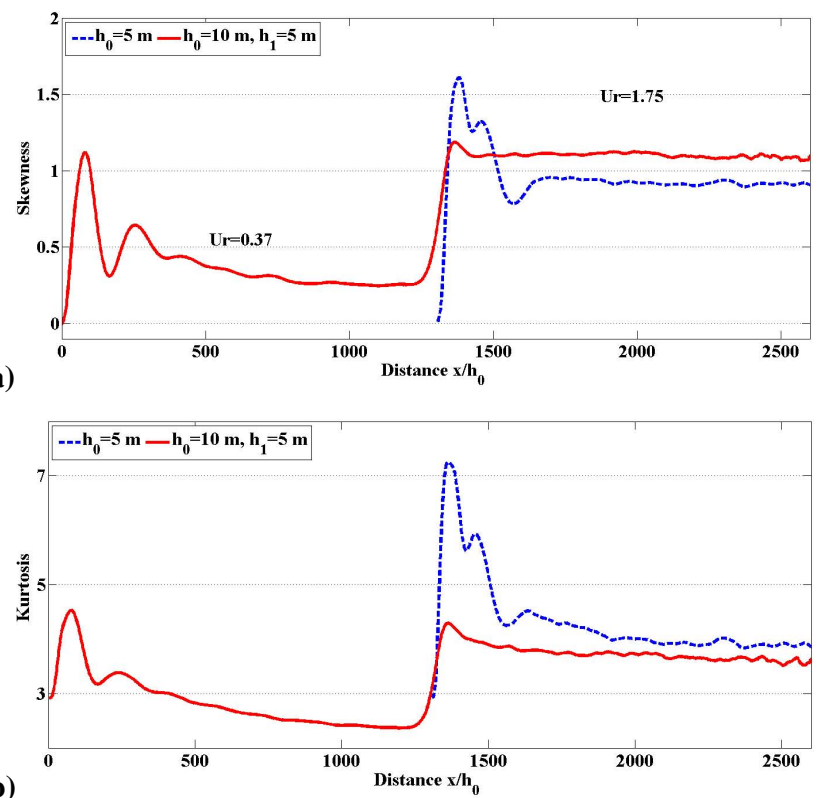

(b)

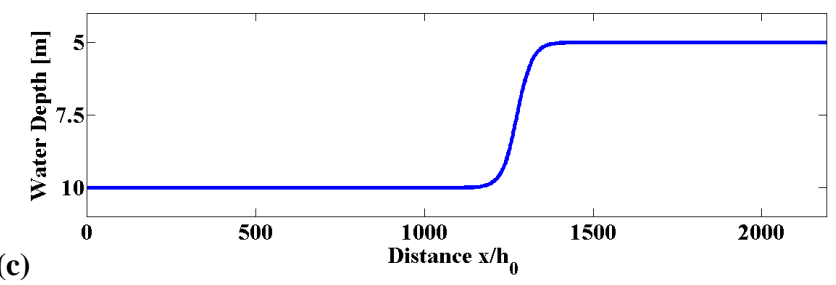

Fig. 2. Variation of the skewness (a) and kurtosis (b) in the water of variable depth with initial value $U r=0.37$ (solid line). The same variation in the water of constant depth $h_{0}=5 \mathrm{~m}$ with $U r=1.75$ (dashed line). Variable depth profile with $L=30 h_{0}$ (c).

parameter equals to 0.37 . Figure $2 \mathrm{a}$ and $\mathrm{b}$ indicates the spatial evolution of the statistical moments, the skewness $\mu_{3}$ and kurtosis $\mu_{4}$, are marked with the red solid line. The variation of the water depth profile is presented in Fig. 2c.

While the depth is constant before the transitional zone, the quasi-stationary state for $\mu_{3}$ and $\mu_{4}$ is achieved (Pelinovsky and Sergeeva, 2006). It is characterized by the (a)

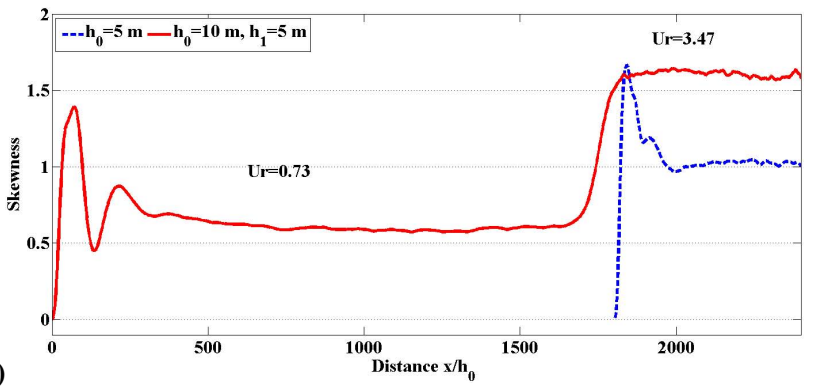

(b)
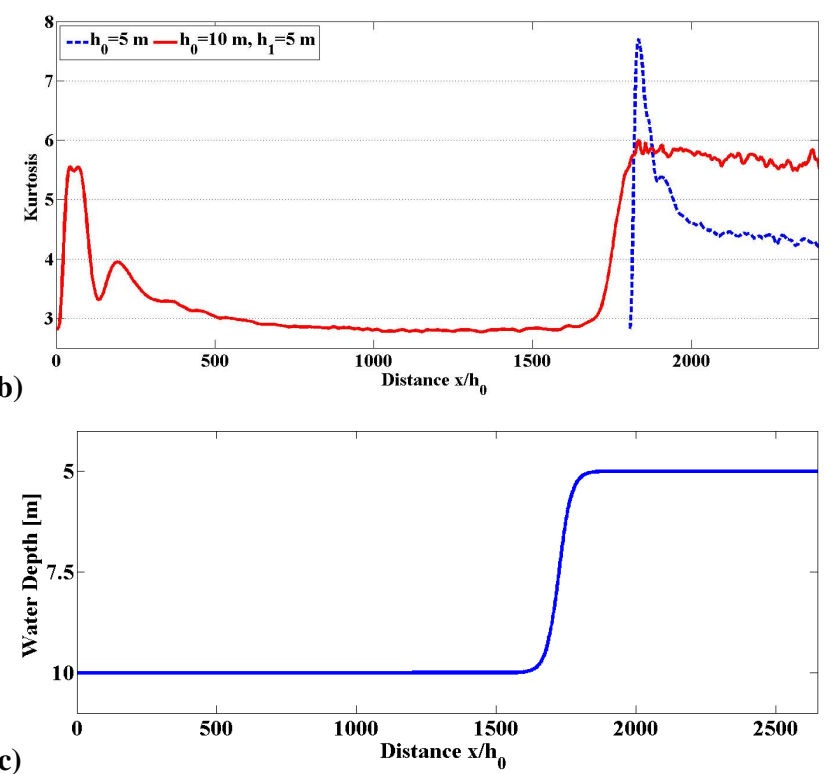

Fig. 3. As in Fig. 1a and $\mathrm{b}$ for $U r=0.73$ and $U r=3.47$. Depth profile with $L=30 h_{0}$ (c).

increase of the wave asymmetry and by the growth of the number of high amplitudes in comparison with the Gaussian distribution; thus, the process deviates far from the Gaussian one. The water depth variation leads to the growth of the wave nonlinearity, and the Ursell parameter grows up to $U r=1.75$ as well. For the new condition, a new steady state can be observed at distances over $x=1500 h_{0}$.

For a comparison, the evolution of random waves with an initially Gaussian-shaped spectrum was considered for the condition of constant depth, $h_{0}=5 \mathrm{~m}$, for the same value of the Ursell parameter, $U r=1.75$. These results were compared with the previous simulations in Fig. $2 \mathrm{a}$ and $\mathrm{b}$ (blue dashed lines correspond to the condition of constant depth). Both statistical moments tend to quite similar values in the two simulations.

An increase of wave steepness $\left(\varepsilon=1 \times 10^{-2}\right)$ leads, as expected, to more significant deviations of skewness and kurtosis from the values of the Gaussian state (Fig. 3). As opposed to the previous case $(U r=1.75)$, the comparison with the simulation for a constant depth in the case of steeper waves $(U r=3.47)$ manifests two different regimes of the steady state at the depth $h=5 \mathrm{~m}$ : a large transition zone 

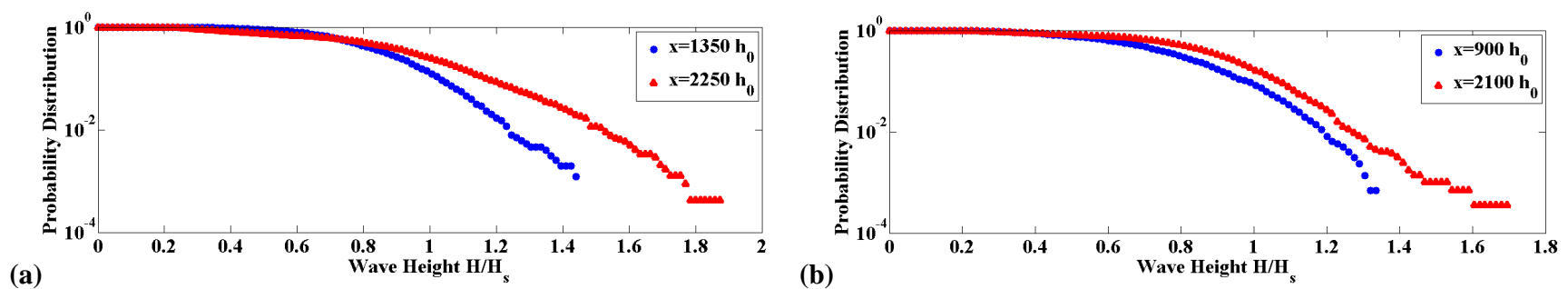

Fig. 4. Exceedance probability distribution function of wave heights scaled by $H_{\mathrm{s}}$ for water depth $h=10 \mathrm{~m}$ (blue circles) and $h=5 \mathrm{~m}$ (red triangles) for two cases of initial wave steepness: $\varepsilon=1 \times 10^{-2}$ (a) and $\varepsilon=5 \times 10^{-3}$ (b).

(a)

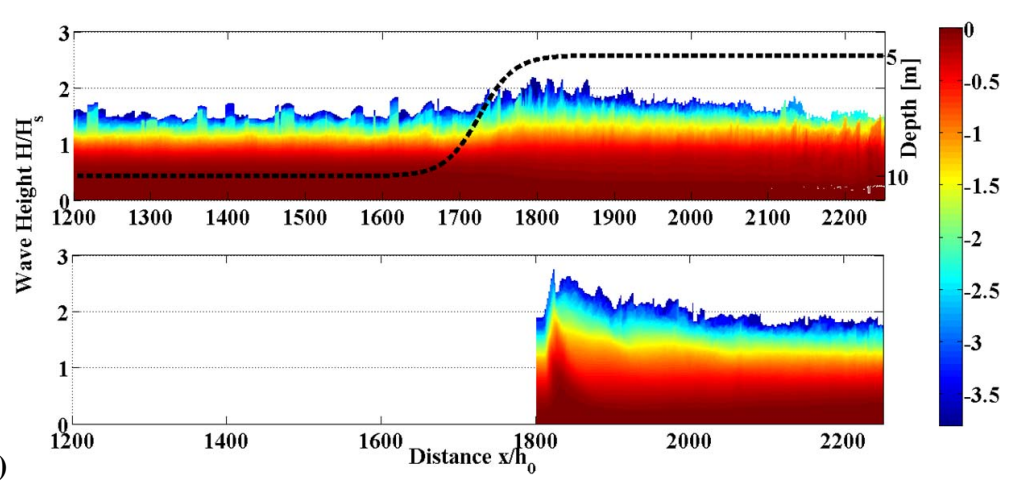

(b)

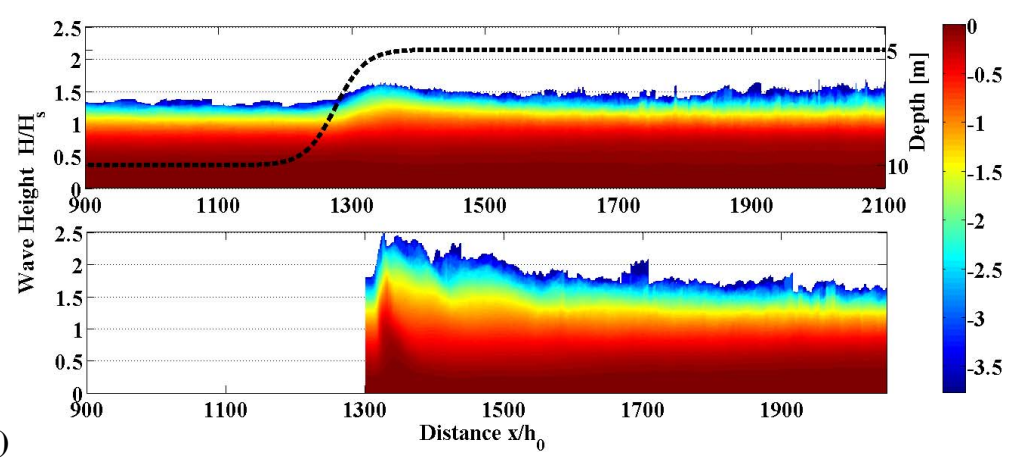

Fig. 5. Spatial diagram of exceedance probability distribution function of wave heights, scaled by $H_{\mathrm{S}}$, in the logarithmic scale for varying depth (upper panel) and constant depth $h=5 \mathrm{~m}$ (lower panel) for two cases of wave steepness, corresponding to $U r=3.5$ (a) and $U r=1.75$ (b).

for a condition of constant depth and a shorter transformation to a steady state for water with depth decrease. The results of calculations of wave propagation over variable depth conditions demonstrate significant increase of the values of kurtosis and skewness compared to the condition of constant depth.

As previously mentioned, the decrease of water depth leads to the increase of nonlinearity and the Ursell parameter as well. It was shown (Figs. 2 and 3) that statistical moments also tend to increase while the water shallows. All this influences the probability distribution of large wave formation and leads to the growth of the tails of distribution function after the transition zone. Figure $4 \mathrm{a}$ and $\mathrm{b}$ demonstrates such behaviour of the probability distribution functions of wave height, scaled by $H_{\mathrm{s}}=4 \sigma$, both for two cases of initial values of wave steepness $\varepsilon=1 \times 10^{-2}$ and $\varepsilon=5 \times 10^{-3}$, where blue circles correspond to the depth of $10 \mathrm{~m}$ and red triangles indicate the probabilities of wave heights in shallower water $(h=5 \mathrm{~m})$.

The spatial variation of the exceedance probability function for wave heights, $\log \left(F\left[H / H_{\mathrm{S}}\right]\right)$, presented in spatial diagrams $\left(H / H_{\mathrm{s}}, x / h_{0}\right)$ of Fig. 5a and b, allows to follow the formation of large waves along the distance for the two cases examined above to be indicated. The profile of the water depth is also given for reference by the dashed line, and the right vertical axis indicates the depth values. For comparison, the variations of the wave height probabilities obtained for the conditions of a constant water depth $h=5 \mathrm{~m}$ and the corresponding Ursell parameters are presented in Fig. 5a and b below the main panels. 


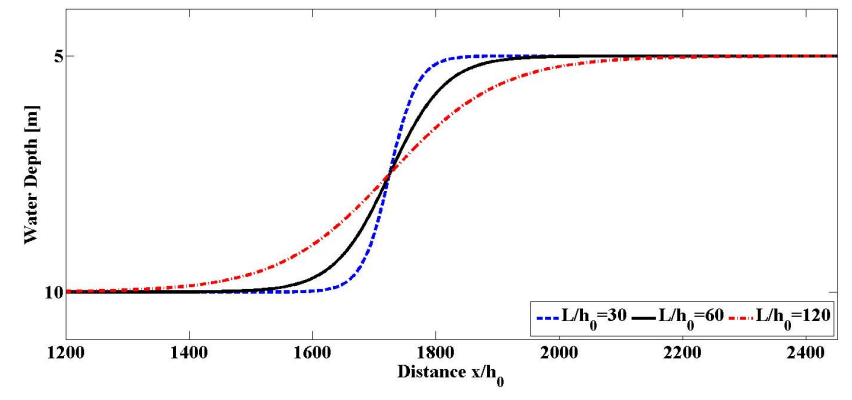

Fig. 6. Depth profile (7) with various length of the transitional zone $L / h_{0}$.

For both sets of the numerical experiments the variation of water depth correlates with the evidence of formation of larger waves, whose heights more than twice exceed the significant wave height, $H_{\mathrm{s}}$, (so-called freak waves). A visible transient zone exists for the probability distribution, which is smaller then one corresponding to the constant water depth case. In the steep wave case ( $U r=3.47$, Fig. 4a) the maximum amplification of wave height is observed when the depth attains a value of $5 \mathrm{~m}$. At the same time, waves of the same heights and even higher occur during wave evolution over water of constant depth for the same conditions of the Ursell parameter (Fig. 5a, the lower panel). In the case of a smaller Ursell parameter $(U r=1.75)$, no such amplification of wave height probability for variable depth case could be observed (Fig. 5b). During the depth jump no freak waves are found (Fig. 5a), and the wave heights do not exceed $2 H_{\mathrm{s}}$, in contrast to the comparative simulation for the constant depth $h=5 \mathrm{~m}$ (Fig. 5a, the lower panel).

To evaluate the influence of the transitional zone length, we consider three values of $L / h_{0}$ (Fig. 6), representing steep and smooth depth profiles. The length of the transitional zone does not influence the quantitative parameters (Fig. 7). At large distances from the zone of the depth transformation, the value of kurtosis seems to slightly decay and tends to one of steady state.

\section{Wave transformation from shallower to deeper water}

An increase of water depth from $5 \mathrm{~m}$ to $10 \mathrm{~m}$ leads to the attenuation of local wave nonlinearity. Nevertheless, the characteristics of the steady state vary significantly. The deviations from the Gaussian statistics descend appreciably. In particular, kurtosis (Fig. 8b) is close to the values typical for the Gaussian sea state $\left(\mu_{4}=3\right)$, and the wave asymmetry (Fig. 8a) becomes less pronounced. The comparison of the statistical parameters with the results obtained for the case of constant depth for the same Ursell parameter (see the dashed line in Fig. 8) demonstrates satisfactory agreement for skewness, and some underestimation of kurtosis for the case of constant depth. (a)
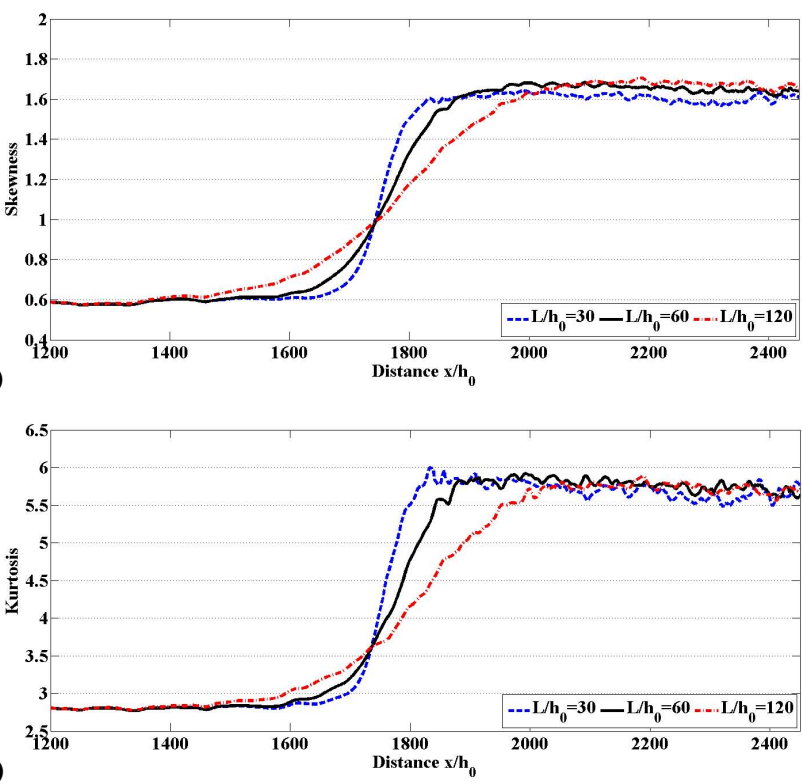

Fig. 7. Transformation of the skewness (a) and the kurtosis (b) for different values of transitional zone $L / h_{0}, h_{0}=10 \mathrm{~m}, U r=3.47$.

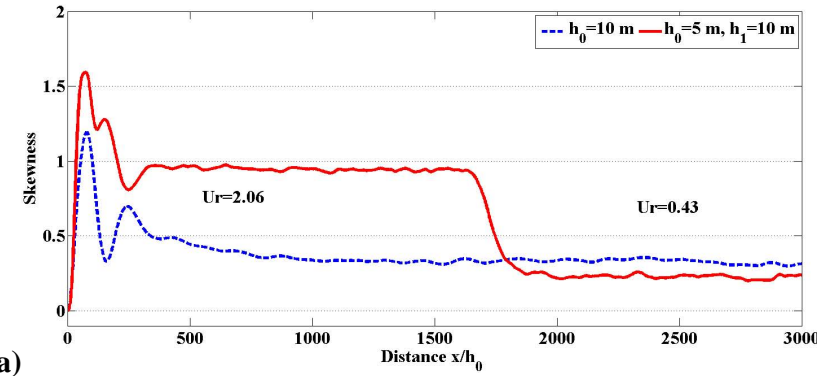

(a)

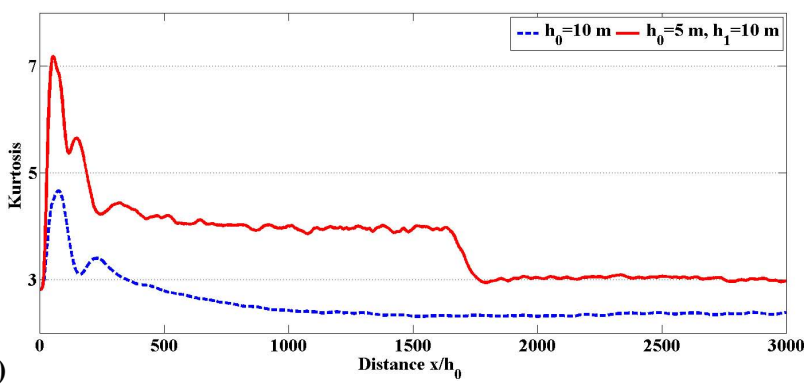

(c)

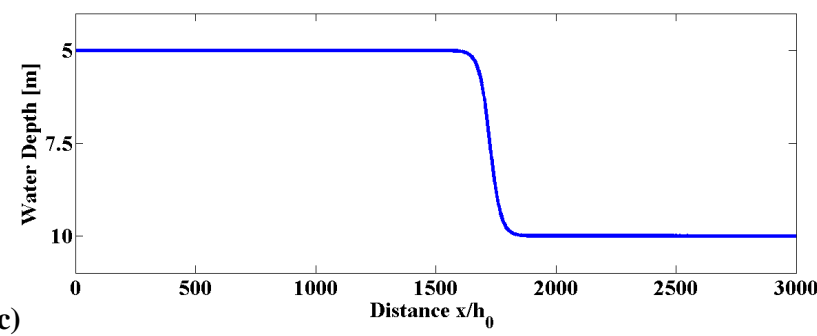

Fig. 8. Variation of skewness (a) and kurtosis (b) in water of variable depth with initial value $U r=2.06$ (solid line). The same variation in water of constant depth $h_{0}=5 \mathrm{~m}$ with $U r=0.43$ (dashed line). Variable depth profile with $L=30 h_{0}$ (c). 


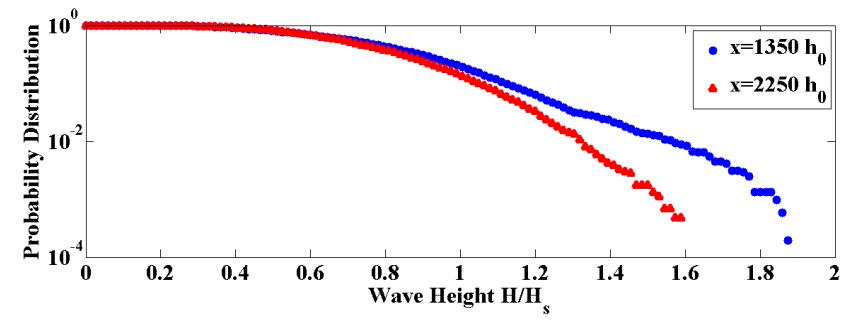

Fig. 9. The exceedance probability distribution function of wave heights scaled by $H_{\mathrm{S}}$ corresponded to $h=5 \mathrm{~m}$ (blue circles) and $h=10 \mathrm{~m}$ (red triangles).

We thus conclude that the probability of extremely high waves decreases when the water becomes deeper, which is demonstrated in Fig. 9, where distribution functions corresponding to $h=10 \mathrm{~m}$ and $h=5 \mathrm{~m}$ are presented.

\section{Conclusions}

The transformation of random (irregular) wave fields in basins of a variable depth is analyzed within the shallow water approximation in the framework of the variablecoefficient Korteweg-de Vries equation. The characteristic amplitude of the surface elevation varies with depth according to Green's law, which follows the theoretical model. Skewness and kurtosis are obtained on the basis of numerical simulations. They are shown to increase when the depth decreases (water shallowing), and the wave state deviates from the Gaussian. The probability of largeamplitude (rogue) waves increases in the transition zone. The characteristics of this process depend on the initial wave steepness (the Ursell parameter), but not on the length of the transitional zone. The results obtained confirm the conclusion made by Zeng and Trulsen (2010) in the framework of the nonlinear Schrodinger equation for narrowbanded wind wave field, that kurtosis and the number of freak waves may significantly differ from the values expected for a flat bottom of a given depth. The same conclusion was arrived at for the representation of the wave field as a low-density "solitonic gas"; the high-amplitude tail of the distribution function increases more significantly when the water becames shallower. If the waves propagates into a deeper water region, the number of freak waves decreases, and the wave field becames closer to the linear one.

Similar effects are believed to exist for the case of shallow-water internal wave fields described by the extended Korteweg-de Vries equation (Holloway et al., 1997, 1999; Grimshaw et al., 2007). In particular, rogue internal waves are predicted theoretically in the framework of the modified Korteweg-de Vries equation, in which the quadratic nonlinear term equals to zero (Grimshaw et al., 2005), and the Gardner equation has a positive cubic nonlinear term (Grimshaw et al., 2010).
Acknowledgements. The authors would like to acknowledge partial support from a RFBR grant 09-05-00204, the President grant MK-6734.2010.5, the State Contract No. 02.740.11.0732 and the RAS Programme "Nonlinear Dynamics". A part of the research was supported by the European Community's Seventh Framework Programme FP7-SST-2008-RTD-1 under grant agreement No. 234175.

Edited by: C. Kharif

Reviewed by: H. B. Branger and another anonymous referee

\section{References}

Didenkulova, I. I., Slunyaev, A. V., Pelinovsky, E. N., and Kharif, C.: Freak waves in 2005, Nat. Hazards Earth Syst. Sci., 6, 10071015, doi:10.5194/nhess-6-1007-2006, 2006.

Didenkulova, I., Pelinovsky, E., and Sergeeva, A.: Runup of long irregular waves on plane beach, in: Extreme Ocean Waves, edited by: Pelinovsky, E. and Kharif, C., Springer, 83-94, 2008.

Didenkulova, I., Pelinovsky, E., and Sergeeva, A.: Statistical characteristics of long waves nearshore, Coast. Eng., 58, 94-102, doi:10.1016/j.coastaleng.2010.08.005, available at: http://dx.doi.org/10.1016/j.coastaleng.2010.08.005, 2011.

Drazin, P.G. and Johnson, R. S.: Solitons: an Introduction, Cambridge Univ. Press, 1996.

Dysthe, K., Krogstad, H. E., and Muller, P.: Oceanic rogue waves, Annu. Rev. Fluid Mech., 40, 287-310, 2008.

Fornberg, B.: A practical guide to pseudospectral methods, Cambridge University Press, 1998.

Grimshaw, R., Pelinovsky, E., Talipova, T., Ruderman, M., and Erdelyi, R.: Short-lived large-amplitude pulses in the nonlinear long-wave model described by the modified Korteweg-de Vries equation, Stud. Appl. Math., 114(2), 189-210, 2005.

Grimshaw, R., Pelinovsky, E., and Talipova, T.: Modeling internal solitary waves in the coastal ocean, Surv. Geophys., 28(4), 273 298, 2007.

Grimshaw, R., Pelinovsky, E., Talipova, T., and Sergeeva, A.: Rogue internal waves in the ocean: long wave model, Eur. Phys. J.-Spec. Top., 185, 195-208, 2010.

Holloway, R., Pelinovsky, E., Talipova, T., and Barnes, B.: A nonlinear model of the internal tide transformation on the Australian North West Shelf, J. Phys. Oceanogr., 27(6), 871-896, 1997.

Holloway, P., Pelinovsky, E., and Talipova, T.: A Generalised Korteweg-de Vries model of internal tide transformation in the coastal zone, J. Geophys. Res., 104(C8), 18333-18350, 1999.

Huntley, D. A., Guza, R. T., and Bowen, A. J.: A universal form for shoreline run-up spectra, J. Geophys. Res., 82(18), 2577-2581, 1977.

Janssen, P. A. E. M.: Nonlinear four wave interaction and freak waves, J. Phys. Oceanogr., 33, 863-884, 2003.

Johnson, R. S.: On the development of a solitary wave moving over an uneven bottom, PCPS-P. Camb. Philol. S., 73, 183-203, 1973.

Kharif, C., Pelinovsky, E., and Slunyaev, A.: Rogue Waves in the Ocean, Springer, 2009

Lavrenov, I.: Wind waves in oceans, Springer-Verlag, 2003.

Liu, P. C.: A chronology of freaque wave encounters, Geofizika, 24(1), 57-70, 2007. 
Mori, N. and Janssen, P. A. E. M.: On kurtosis and occurrence probability of freak waves, J. Phys. Oceanogr., 36, 1471-1483, 2006.

Nakoulima, O., Zahibo, N., Pelinovsky, E., Talipova, T., and Kurkin, A.: Solitary wave dynamics in shallow water over periodic topography, Chaos, 15, 037107, doi:10.1063/1.1984492, 2005.

Novikov, S., Manakov, S. V., Pitaevskii, L. P., and Zakharov, V. E.: Theory of Solitons: the Inverse Scattering Method, Consultants Bureau, New York, 1984.

Onorato, M., Osborne, A. R., Serio, M., Cavaleri, L., Brandini, C., and Stansberg, C. T.: Extreme waves, modulational instability and second-order theory: wave flume experiments on irregular waves, Eur. J. Mech. B-Fluid., 25, 586-601, 2006.

Ostrovsky, L. A. and Pelinovsky, E. N.: Wave transformation of the surface of a fluid of variable depth, Izvestiya, Atmos. Oceanic Phys., 6(9), 552-555, 1970.

Pelinovsky, E. N.: On the soliton evolution in inhomogeneous media, Applied Mechanics and Technical Physics, 6, 80-85, 1971.

Pelinovsky, E. and Sergeeva (Kokorina), A.: Numerical modeling of the KdV random wave field, Eur. J. Mech. B-Fluid., 25, 425434, 2006

Salupere, A., Maugin, G. A., Engelbrecht, J., and Kalda, J.: On the KdV soliton formation and discrete spectral analysis, Wave Motion, 123, 49-66, 1996.
Salupere, A., Peterson, P., and Engelbrecht, J: Long-time behaviour of soliton ensembles. Part 1 - Emergence of ensembles, Chaos Soliton. Fract., 14, 1413-1424, 2002.

Salupere, A., Peterson, P., and Engelbrecht, J.: Long-time behaviour of soliton ensembles. Part 2 - Periodical patterns of trajectories, Chaos Soliton. Fract., 15, 29-40, 2003 a.

Salupere, A., Peterson, P., and Engelbrecht, J.: Long-time behavior of soliton ensembles, Math. Comput. Simulat., 62, 137-147, 2003b.

Shemer, L. and Sergeeva, A.: An experimental study of spatial evolution of statistical parameters in a unidirectional narrowbanded random wave field, J. Geophys. Res., 114, C01015, doi:10.1029/2008JC005077, 2009.

Shemer, L., Sergeeva, A., and Slunyaev, A.: Applicability of envelope model equations for simulation of narrow-spectrum unidirectional random field evolution: experimental validation, Phys. Fluids, 22, 016601, 1-9, doi:10.1063/1.3290240, 2010.

Tappert, F. D. and Zabusky, N. J.: Gradient-induced fission of solitons, Phys. Rev. Lett., 27, 1774-1776, 1971.

Zakharov, V. E.: Kinetic equation for solitons, Soviet JETP, 60, 993-1000, 1971.

Zeng, H. and Trulsen, K.: Can non-uniform bathymetry provoke freak waves?, Geophysical Research Abstracts, 12, EGU2010 8306, EGU General Assembly 2010, 2010. 